\title{
A simple lattice Boltzmann model for conjugate heat transfer
}

\author{
S. Chen ${ }^{* 1,2}$, Y.Y. Yan ${ }^{2}$, W. Gong ${ }^{2}$ \\ 1. Institute for Modelling and Simulation in Fluodynamics, Nanoscience and \\ Industrial Mathematics "Gregorio Millán Barbany", Universidad Carlos III de \\ Madrid, Leganes 28911, Spain \\ 2. Faculty of Engineering, The University of Nottingham, University Park, \\ Nottingham NG7 2RD, UK \\ * Corresponding author. Faculty of Engineering, University of Nottingham. E-mail \\ address: ezzsc2@exmail.nottingham.ac.uk
}

\begin{abstract}
In this paper a lattice Boltzmann (LB) model is proposed for conjugated heat transfer research. Through taking the most advantages of the standard LB method, the present model can remedy the shortcomings of the available related LB models via a simple way and meanwhile a number of intrinsic advantages of the standard LB method are preserved. It does not require any specific treatment dependent on interface topology and independent from the choice of lattice model. Moreover, it can be used for unsteady problems with complicated and time dependent interfaces. The accuracy and reliability of the present model are validated by three nontrivial benchmark tests. The good agreements between the present numerical prediction and available open data demonstrate the applicability of the present model for complicated conjugated heat transfer problems. Finally, the present model could be extended to some other important areas straightforwardly, such as fluid-solid phase change modeling.
\end{abstract}

Key words: Lattice Boltzmann method; conjugate heat transfer; heterogeneous media

\section{Introduction}

To meet a diversity of purposes of practical applications, almost all realistic systems are constituted by heterogeneous/composite media. Due to the balance between cost and performance, in the manufacturing industry, the popularly used heterogeneous/composite media are made up of several material 
layers with different thermodynamic properties. Consequently, complicated interfaces between different material layers are commonly found in realistic systems. In the area of heat transfer research, these interfaces are classified as conjugate problems where the so-called conjugate boundary condition is applicable[1]. Namely, on the interfaces, the temperature profile and heat flux both should be continuous, according to the local thermodynamic equilibrium assumption and the law of energy conservation. From the viewpoint of scientific computing, the challenge of a conjugate problem may come from solving a strongly "coupled" interface domain which is subject to Dirichletand Neumann-like boundary restriction at the the same time (but please bear in mind that a conjugate boundary condition is neither a standard Dirichlet/Neumann boundary condition nor a combination of them). No doubt, how to accurately and efficiently solve a conjugate problem has been an important topic in heat transfer research $[2,3]$. Especially, to the popularly used numerical techniques, their algorithmic complication will be dramatically enhanced for a conjugate problem with a complex interface [3].

During the past three decades, the lattice Boltzmann (LB) method has attracted increasing attention due to its some intrinsic advantages, such as relatively easy treatment of complicated geometry, high parallel computing efficiency and capturing interaction between different phases/components at a mesoscopic level [4]. Especially, as it is a particle-based numerical solver, the LB method can guarantee, automatically, the continuity of a certain macroscopic quantity and of its flux across an arbitrary interface within the investigated domain, if the macroscopic quantity and its flux can be recovered from the zeroth- and first-order moment of the corresponding pseudo-particle distribution function, respectively, in the LB framework. This feature is a potential great advantage for conjugate heat transfer research as in the LB framework one need not explicitly treat the topology of the interface where the conjugated boundary condition should be strictly satisfied. Wang et al. [5] perhaps are the pioneers to utilize this upside. They constructed a LB model for conjugate heat transfer simulation without any explicit treatment on interfaces and observed that the LB approach could solve conjugate heat transfer problems more efficiently than traditional numerical techniques, benefitting from such intrinsic advantage of the LB scheme. Unfortunately, meanwhile their model suffers from three obvious shortcomings: (1) in their model any interface has to be located exactly on the middle point between two lattice grids; (2) the model can work well only in steady scenarios; and (3) the specific heat capacity should be homogenous within the investigated domain even the domain is filled with heterogenous media. In order to remedy the latter two defects, the same research group [6] proposed another LB model. Although their new model reached their expectation successfully, the major superiority of the LB method for conjugate problem modeling was lost. Namely, in Ref.[6] the interfaces between heterogenous media should be handled explicitly. As a result, their discussions were limited for the cases restricted by straight-interface ge- 
ometry [6]. To model conjugate problems with arbitrary interfaces, Li et al.[7] and Le et al.[8] designed an improved scheme, respectively. However, as interpolation and/or extrapolation are required, these schemes, especially the former, are complicated and lose the intrinsic advantage of local computing of the standard LB method. Moreover, in many scenarios the normal heat flux across an interface can be hardly evaluated, which limits the applicable range of these schemes. An immersed-boundary-like LB scheme was also proposed by $\mathrm{Hu}$ et al.[9] to deal with conjugate heat transfer across curved interfaces. In their work curved interfaces were approximated by zigzags and one had to assume that the ratio of thermal conductivities and the ratio of thermal diffusivities should be identical [9]. The former treatment will change the hydrodynamic characteristics of the investigated object and the latter one is hardly satisfied by realistic systems. In addition, how to choose a suitable delta function is a crucial issue for the immersed boundary method, which will influence numerical accuracy critically. Unfortunately, it is a challenge to determine an appropriate multidimensional delta function[10].

In order to fully utilize the intrinsic advantage of the LB method to model conjugate heat transfer with complicated interfaces, which is not achieved by the aforementioned efforts [5-9], Karani and Huber [11] proposed a different strategy. They pointed out, for the first time, the root why the standard LB method can not work for unsteady conjugate heat transfer simulation is the spatial variation of the heat capacitance (the product of the density and specific heat capacity) across interfaces can not be modelled appropriately by the standard LB scheme. Accordingly, the authors designed a source term, which was added into the standard LB evolving equation, to correct the errors generated by the standard LB method. Different from the schemes proposed in Refs. [6-9], their strategy looks avoiding any specific treatment depending on the interface topology. However, after a careful survey, one can find out that the determination of the average heat capacitance (in their source term) on interfaces still relies on the interface topology implicitly. Furthermore, it was observed that their strategy lacks of mathematical rigor in differentiating the piecewise capacitance constant function [12]. As the key to extend the standard LB scheme to conjugate heat transfer research is to capture the spatial variation of the heat capacitance across interfaces accurately, Huang and $\mathrm{Wu}$ [13] claimed their LB model for phase change modelling could be adopted, after a minor modification, for conjugate heat transfer simulation, without any explicit treatment on interfaces. However, in their model [13] only the spatial variation of the specific heat capacity, rather than the heat capacitance, can be modelled, so their claim is tenable only when the density is identical over the whole investigated domain. It is a too strong restriction to simulate conjugate problems in realistic systems. Recently, a enthalpy-based LB model for unsteady conjugate heat conduction between solid heterogeneous media was published [12]. A normalized sensible enthalpy, rather than temperature used in previous studies $[5-9,11]$, was adopted to construct their evolving equation 
[12]. In order to offset the additional terms brought by the introduction of the normalized sensible enthalpy, a source term including temporal difference was added accordingly. For simulation of conjugated heat conduction across solid-solid arbitrary interfaces, no special treatment on interfaces was required in their scheme[12]. Such strategy can remedy the shortcoming caused by the determination of the average heat capacitance (in the source term) on interfaces, which has to be addressed by the LB scheme developed in Ref.[11], but with a cost of degrading the numerical accuracy (the LB model in Ref.[12] is first-order temporal accuracy due to the introduction of the source term while the temporal accuracy of the standard LB method is second-order). Moreover, their strategy will become very complicated if it is extended for conjugate fluid-solid problems as where one has to calculate spatial gradients of enthalpy flux emerging in the source term. Numerical accuracy and stability both will be hampered seriously. In addition, the intrinsic advantage of local computing of the standard LB method will be damaged by the spatial gradients in their source term.

In this work, we propose a simple LB scheme for conjugate heat transfer research. It does not need any specific treatment on arbitrary interfaces and meanwhile can keep the intrinsic advantages of the standard LB method, such as local computing. Therefore, the present scheme can remedy the shortcomings of the available relevant models for conjugate heat transfer simulation. Moreover, it can be extended to model solid-liquid phase change problems straightforwardly. The rest of this paper is organized as follow: in section 2 we present this simple LB model together with a comparison analysis to previous efforts and the corresponding numerical validation is conducted in Section 3 , followed by a conclusion on this work. Although in the present work we take the single-relaxation-time (SRT) LB model as an example to show how to establish a simple LB approach for conjugate heat transfer simulation, its multiple-relaxation-time (MRT) counterpart is also easy to be constructed, following the MRT template used in Ref.[8].

\section{LB model for conjugated heat transdfer}

\subsection{Macroscopic governing equation for conjugate heat transfer}

For conjugate heat trnasfer modeling, the best choice is the conservation form of energy equation which reads [11]:

$$
\partial_{t} \rho C_{p} T+\nabla_{\alpha} \rho C_{p} T u_{\alpha}=\nabla_{\alpha} \lambda \nabla_{\alpha} T
$$


where $\rho, u_{\alpha}$ and $T$ are the density, velocity and temperature of working media, respectively. In addition, $\lambda$ and $C_{p}$ denote the thermal conductivity and constant pressure specific heat capacity.

As analyzed in detail by Refs.[11-13], however, the recovered macroscopic energy governing equation by the standard LB method reads:

$$
\partial_{t} \rho C_{p} T+\nabla_{\alpha} \rho C_{p} T u_{\alpha}=\nabla_{\alpha} \kappa \nabla_{\alpha} \rho C_{p} T
$$

where $\kappa=\lambda /\left(\rho C_{p}\right)$ is the thermal diffusivity of working media. Consequently, across any arbitrary interface within the investigated domain, in the framework of the standard LB method, only the following equalities can be guaranteed:

$$
\begin{aligned}
T_{+} & =T_{-} \\
n_{\alpha}\left[\kappa \nabla_{\alpha} \rho C_{p} T\right]_{+} & =n_{\alpha}\left[\kappa \nabla_{\alpha} \rho C_{p} T\right]_{-}
\end{aligned}
$$

where $n_{\alpha}$ is normal to the interface, and [ $]_{+}$and [ ]- indicate the parameters at each side of the interface.

Compared with the conjugate boundary restriction $[2,3]$ :

$$
\begin{aligned}
T_{+} & =T_{-} \\
n_{\alpha}\left[\lambda \nabla_{\alpha} T\right]_{+} & =n_{\alpha}\left[\lambda \nabla_{\alpha} T\right]_{-}
\end{aligned}
$$

there is an obvious difference between Eq.(4) and Eq.(6). Only when $\left[\rho C_{p}\right]_{+}=$ $\left[\rho C_{p}\right]_{-}$or at the steady status, Eq.(4) can approximate to Eq.(6) exactly. Consequently, for the LB approach, the key to accurately model conjugate problems is to recover the diffusion term in the energy equation exactly (i.e. the term at the right hand of Eq. (1)).

\subsection{Derivation of the present $L B$ model for conjugate heat transfer modelling}

As revealed by Karani and Huber [11], the key to recover the diffusion term in the energy equation Eq. (1) exactly is to treat the spatial variation of the heat capacitance appropriately. In the present work, inspired by Ref.[12], a sensible-enthalpy-like quantity $h^{*}=\left(\rho C_{p}\right)_{0} T$ is introduced, where $\left(\rho C_{p}\right)_{0}$ is the reference heat capacitance. With the aid of $h^{*}$, Eq. (1) can be transformed to (please refer to the Appendix for the detail):

$$
\partial_{t} h^{*}+\nabla_{\alpha} h^{*} u_{\alpha}=\nabla_{\alpha} \frac{\lambda}{\rho C_{p}} \nabla_{\alpha} h^{*}-\frac{\lambda}{\left(\rho C_{p}\right)_{0}} \nabla_{\alpha} h^{*} \nabla_{\alpha} \frac{1}{\sigma}-\frac{h^{*}}{\sigma} u_{\alpha} \nabla_{\alpha} \sigma
$$

where $\sigma=\frac{\rho C_{p}}{\left(\rho C_{p}\right)_{0}}$ is the ratio of heat capacitance. If the heat capacitance is homogenous (namely, $\nabla_{\alpha} \frac{1}{\sigma}=\nabla_{\alpha} \sigma=0$ ), the last two terms in Eq.(7) will 
automatically vanish.

Equation (7) is a standard advection-diffusion equation respect to $h^{*}$, with a source term $S=-\frac{\lambda}{\left(\rho C_{p}\right)_{0}} \nabla_{\alpha} h^{*} \nabla_{\alpha} \frac{1}{\sigma}-\frac{h^{*}}{\sigma} u_{\alpha} \nabla_{\alpha} \sigma$. It can be solved by the following LB scheme $[4,11]$ :

$$
g_{j}\left(x_{\alpha}+e_{j \alpha} \Delta t, t+\Delta t\right)-g_{j}\left(x_{\alpha}, t\right)=-\frac{1}{\tau}\left[g_{j}\left(x_{\alpha}, t\right)-g_{j}^{(e q)}\left(x_{\alpha}, t\right)\right]+\omega_{j} \Delta t S(8)
$$

where $\omega_{j}$ and $\Delta t$ represents the weight coefficients and dimensionless time step, and $e_{j}$ and $g_{j}\left(x_{\alpha}, t\right)$ denotes the discrete velocity direction and the corresponding pseudo-particle distribution function at the lattice grid $x_{\alpha}$. As discussed in previous research $[8,15]$, for an advection-diffusion equation, a "simpler" lattice model with fewer discrete velocity directions can be used to improve computational efficiency, for example a D2Q5 lattice model for twodimensional problem[8]. However, please bear in mind that the present scheme is independent from the choice of lattice model.

In Eq. (8) $\tau$ is the dimensionless relaxation time and it satisfy the relationship:

$$
\kappa=\frac{\lambda}{\rho C_{p}}=c_{s}^{2}(\tau-0.5) \Delta t
$$

where $c_{s}$ is the speed of sound in the LB framework $[4,15]$.

The equilibrium distribution in Eq.(8) reads[15]

$$
g_{j}^{(e q)}=\omega_{j} h^{*}\left(1+\frac{e_{j \alpha} u_{\alpha}}{c_{s}^{2}}\right)
$$

and the local temperature can be obtained through the zeroth-order moment of $g_{j}\left(x_{\alpha}, t\right)$ :

$$
T=\frac{1}{\left(\rho C_{p}\right)_{0}} \sum_{j} g_{j}
$$

In succession, how to evaluate the source term $S$ appropriately becomes a critical step to guarantee the advantage of local computing of the standard LB method. According to Eq.(13) in Ref.[11], one can get

$$
-\frac{\lambda}{\left(\rho C_{p}\right)_{0}} \nabla_{\alpha} h^{*}=-\sigma \frac{\lambda}{\rho C_{p}} \nabla_{\alpha} h^{*}=\sigma\left(1-\frac{1}{2 \tau}\right) \sum_{j}\left[g_{j}\left(x_{\alpha}, t\right)-g_{j}^{(e q)}\left(x_{\alpha}, t\right)\right] e_{j \alpha}
$$


In addition, in the LB framework there is an approximation [4]:

$$
\nabla_{\alpha} \phi\left(x_{\alpha}\right)=\frac{1}{c_{s}^{2} \Delta t} \sum_{j} \omega_{j} \phi\left(x_{\alpha}+e_{j \alpha} \Delta t\right) e_{j \alpha}+O\left(\Delta t^{2}\right)
$$

where $\phi\left(x_{\alpha}\right)$ is a certain scalar quantity at the lattice grid $x_{\alpha}$.

With the aid of Eq.(13), we can replace $\nabla_{\alpha} \sigma$ and $\nabla_{\alpha} \frac{1}{\sigma}$ in the source term in Eq.(8) by

$$
\nabla_{\alpha} \sigma\left(x_{\alpha}\right)=\frac{1}{c_{s}^{2} \Delta t} \sum_{j} \omega_{j} \sigma\left(x_{\alpha}+e_{j \alpha} \Delta t\right) e_{j \alpha}
$$

and

$$
\nabla_{\alpha} \frac{1}{\sigma}\left(x_{\alpha}\right)=\frac{1}{c_{s}^{2} \Delta t} \sum_{j} \omega_{j} \frac{1}{\sigma}\left(x_{\alpha}+e_{j \alpha} \Delta t\right) e_{j \alpha}
$$

Consequently, the source term $S$ can be written as

$$
\begin{array}{r}
S=\frac{1}{c_{s}^{2} \Delta t} \sigma\left(1-\frac{1}{2 \tau}\right) \sum_{j}\left[g_{j}\left(x_{\alpha}, t\right)-g_{j}^{(e q)}\left(x_{\alpha}, t\right)\right] e_{j \alpha} \sum_{j} \omega_{j} \frac{1}{\sigma}\left(x_{\alpha}+e_{j \alpha} \Delta t\right) e_{j \alpha} \\
-\frac{1}{c_{s}^{2} \Delta t} \frac{h^{*}}{\sigma} u_{\alpha} \sum_{j} \omega_{j} \sigma\left(x_{\alpha}+e_{j \alpha} \Delta t\right) e_{j \alpha}
\end{array}
$$

One can observe that in the present work the calculation of $S$ is a completely local operation. Away from interfaces, the output of Eq.(16) becomes zero since there is no spatial variation of the heat capacitance in a homogenous material layer, consistent to the above statement.

As the reference heat capacitance $\left(\rho C_{p}\right)_{0}$ is a constant over the whole domain, divided by $\left(\rho C_{p}\right)_{0}$, Eq. (7) can be transformed to:

$$
\partial_{t} T+\nabla_{\alpha} T u_{\alpha}=\nabla_{\alpha} \frac{\lambda}{\rho C_{p}} \nabla_{\alpha} T-\frac{\lambda}{\left(\rho C_{p}\right)_{0}} \nabla_{\alpha} T \nabla_{\alpha} \frac{1}{\sigma}-\frac{T}{\sigma} u_{\alpha} \nabla_{\alpha} \sigma
$$

It is obvious that for homogeneous media Eq. (17) will reduce to the popularly used macroscopic governing equation for temperature field. The additional terms are the correction when the investigated domain consists of heterogeneous media. With the aid of Eq. (17), one can observe that the source term proposed in Ref.[11] is incorrect, which has also been pointed out by $\mathrm{Hu}$ et al.[9] but without detailed explanation. According to Eq.(17), it is clear that the term for convective heat flux in Ref.[11] is wrong.

In the present work, Eq.(7) is referred to as an "enthalpic" formulation and Eq. (17) is its "temperature" counterpart. The advantage of the "enthalpic" form Eq.(7) is it can be extended straightforwardly to model solid-liquid phase 
change problems as in such research area the enthalpy-base method has received increasing attention due to its simplicity $[9,17]$. It will be discussed in detail in our future work as it is beyond the scope of the present work.

Compared with previous efforts [6-9], the present model does not need special treatments on the topology of the interfaces between heterogeneous media. Compared with Ref.[5], the present model can model unsteady conjugate heat transfer with complicated interfaces via a simple way. Compared with Karani and Huber's model [11], the present work avoids the puzzle of determining the average heat capacitance on interfaces. Compared with Ref.[13], the present work breaks the limitation of identical density over the whole investigated domain. Compared with the model designed in Ref. [12], the present model keeps the intrinsic advantage of local computing of the standard LB method, besides numerical stability and accuracy. Therefore, the present model realizes to model conjugate problems through taking the most intrinsic advantages of the LB method. Meanwhile, the present model possesses a number of attracting advantages, such as its capablity to model conjugate heat transfer in media with evolving microstructures where interfaces and thermophysical properties change over time (please see the Appendix for the detail).

Finally, if there are internal heat resources in the investigated domain, another source term that represents the internal heat resources should be added into Eq. (8) accordlingly, as shown in Refs.[11,15]. It will be illustrated in detail through the third validation benchmark case below.

\section{Numerical validation}

In order to validate the present model, three non-trivial benchmark tests are adopted. The first one is transient heat conduction in three-layered stratified media [12] which can validate the present model's accuracy for unsteady conjugate problems. The second one is conjugate mixed convection heat transfer in a lid-driven enclosure with thick bottom wall[18], which can check the capability of the present model to conserve conductive and advective heat flux simultaneously on interfaces as in which thermal fluid flow is not always parallel to the interface. The last benchmark test is magnetohydrodynamic (MHD) mixed convection with Joule heating in a lid-driven cavity which contains a heat conducting horizontal circular cylinder [19]. It can demonstrate the reliability of the present model for curved interfaces embeded in complicated fluid and heat flow. The D2Q9 lattice is used for solving flow field and the D2Q5 lattice is employed to compute energy field, similar with our previous work [15]. The LB scheme for fluid flow simulation and boundary treatment used in our previous work $[15,16]$ is adopted here again. 


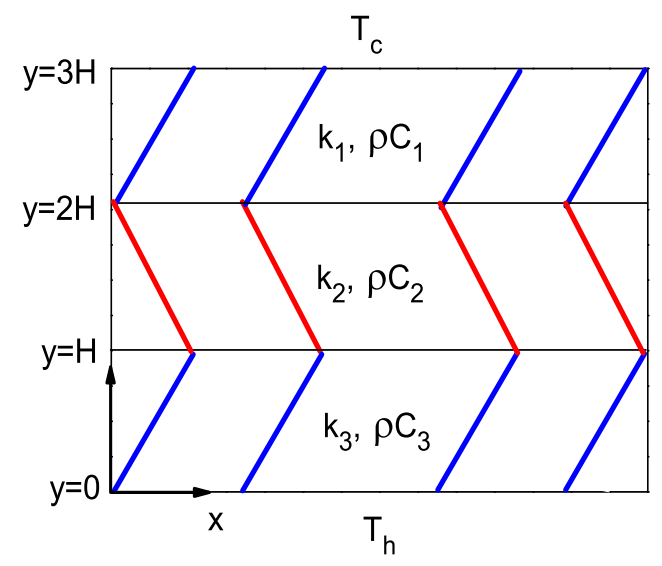

Fig. 1. Schematic configuration of three-layered stratified media.

\subsection{Transient heat conduction in three-layered stratified media}

The transient heat conduction in three-layered stratified media investigated by Ref.[12] is a typical issue in heat transfer research. The schematic configuration is depicted by Fig. 1 . At time $t=0$, the temperature over the whole domain is identical, as $T_{c}=0$. Since $t>0$, the temperature on the downside is elevated and fixed at $T_{h}=1.0$. The vertical sides of the investigated domain both are adiabatic. The thermophysical properties of each layer read [12]: $\lambda_{1}=\lambda_{3}=1$, $\lambda_{2}=0.1,\left(\rho C_{p}\right)_{1}=\left(\rho C_{p}\right)_{3}=1.0$ and $\left(\rho C_{p}\right)_{2}=2.0$. The same as Ref.[12], a grid resolution $100 \times 300$ is employed.

Figure 2 illustrates the temperature profiles along the vertical central line at different instants until the steady status obtained by the present model, compared with the solutions given in Ref.[12] (please refer to Fig. 3 therein). The present results agree well with the data in Ref.[12], which demonstrates the present model's applicability for unsteady conjugate problems.

\subsection{Conjugate mixed convection heat transfer in a lid-driven enclosure with thick bottom wall}

Figure 3 shows the schematic configuration of lid-driven enclosure with thick bottom wall investigated in Ref.[18]. The square cavity is $H \times H$ filled with fluid. The vertical walls both are adiabatic. The solid bottom wall with a thickness $h$ is heated by a fixed high temperature $T_{h}=1$ while the moving top lid is cooled by a constant temperature $T_{c}=0$. The gravity $g$ is downward. The boundary conditions are illustrated by Fig.3. The Richardson number $R i$, which describes the ratio between buoyant convection term and force convection term, is unity. In the present work $h / H=0.1$ and the ratio of thermal 


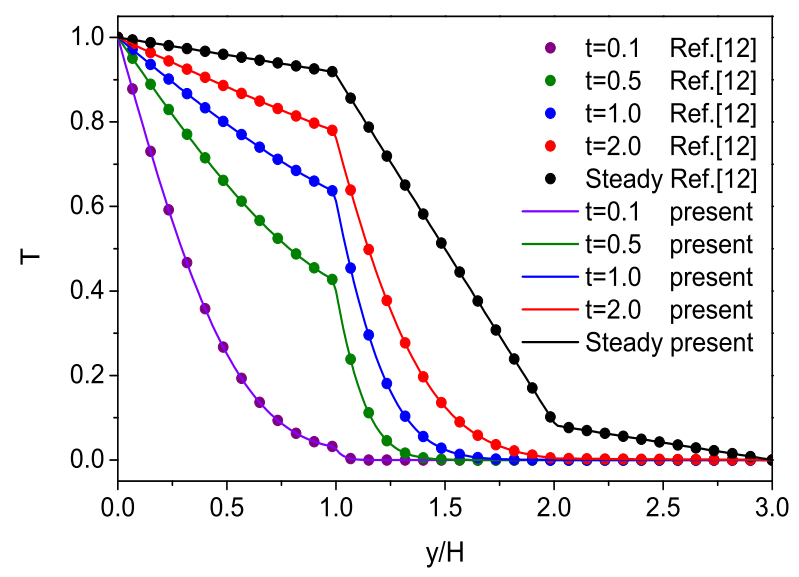

Fig. 2. Temperature profiles along the vertical central line at various instants: solid lines-present results, dots-solutions in Ref.[12].

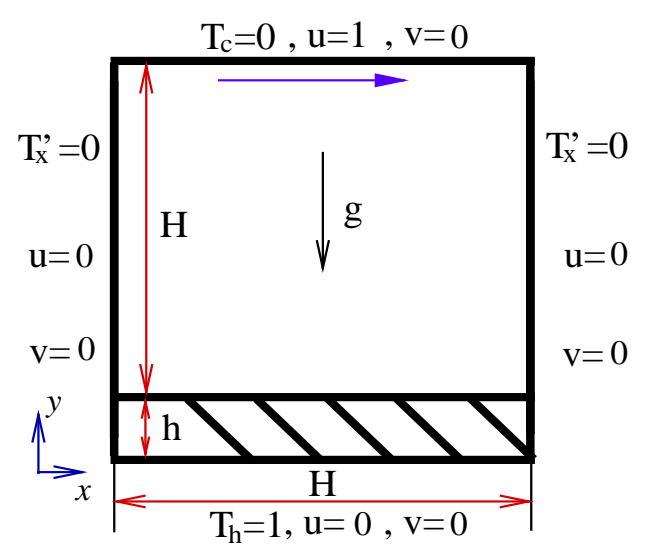

Fig. 3. Schematic configuration of lid-driven enclosure with thick bottom wall.

conductivity between fluid and solid thick bottom wall is $k=\lambda_{f} / \lambda_{s}=0.1,1$ and 10. The detailed description, such as the governing equations, please refer to Ref.[18]. A grid resolution $100 \times 110$ is adopted.

Figures 4-6 plots the streamlines and isotherms at various $k$. With a low $k$ (e.g. $k=0.1$ ), temperature gradient in fluid is large due to its low thermal conductivity. Against $k$ increasing, fluid becomes colder and temperature is distributed inside the solid bottom wall owing to the insulation-like effect of the thick bottom wall. The fluid at the top-right corner of the cavity is always the coldest over the whole domain. The observation is in good agreement with that reported by Ref.[18] (please refer to Figs. 3-5 therein).

The profile of $y$-component velocity along the horizontal central line of the square cavity is depicted by Fig. 7. One can observe that a higher $k$ will prevent the fluid motion. It agrees with the conclusion in Ref.[18] that flow strength was decreased against $k$ increasing. 


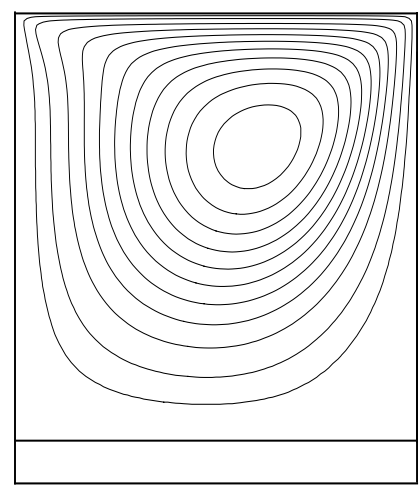

(a)

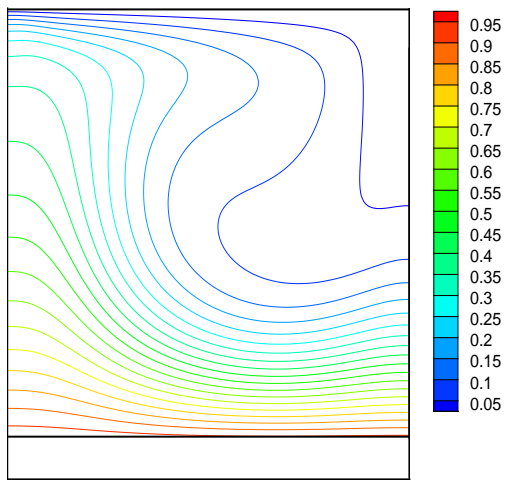

(b)

Fig. 4. (a) streamlines and (b)isotherms for $h / H=0.1, k=0.1$ and $R i=1$.

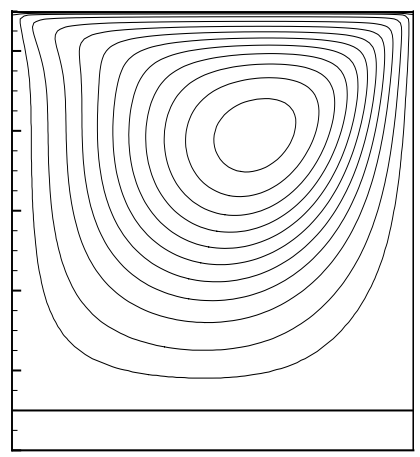

(a)

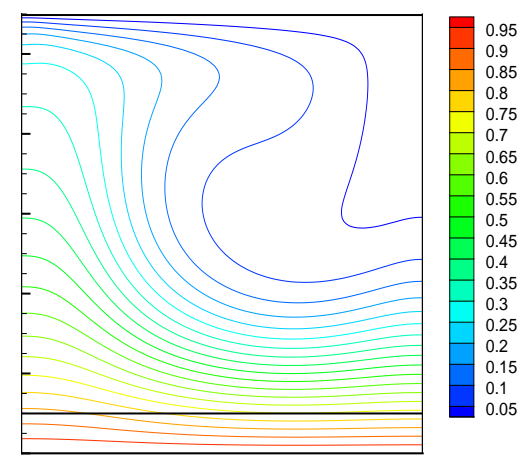

(b)

Fig. 5. (a) streamlines and (b)isotherms for $h / H=0.1, k=1$ and $R i=1$.

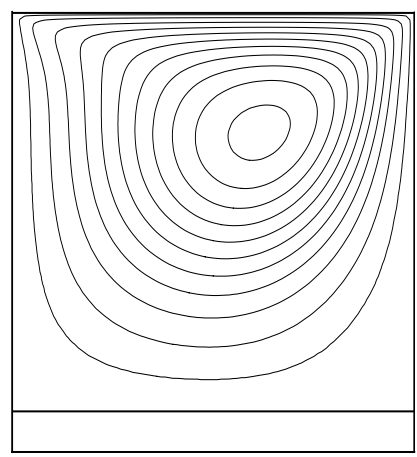

(a)

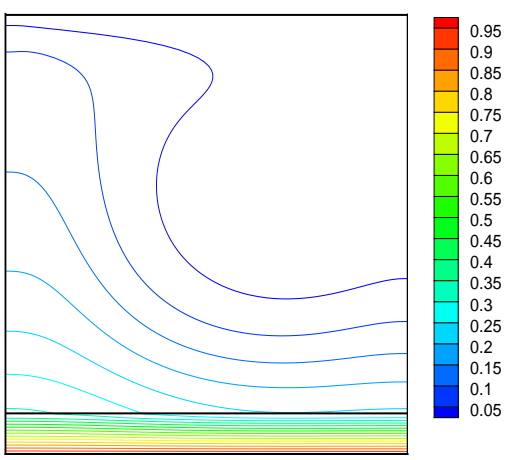

(b)

Fig. 6. (a) streamlines and (b)isotherms for $h / H=0.1, k=10$ and $R i=1$. 


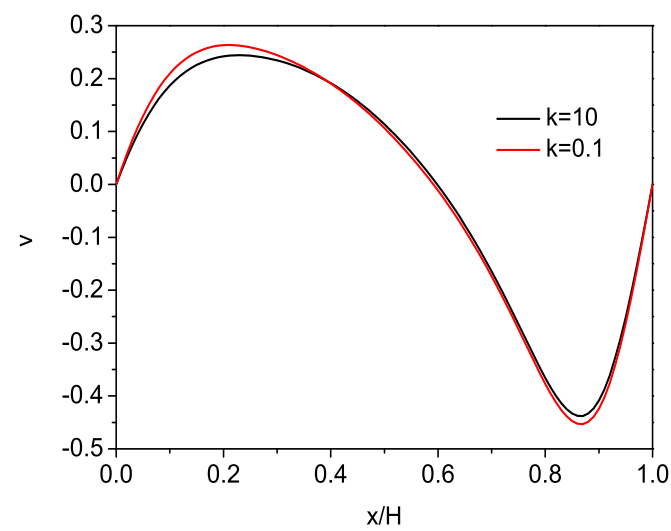

Fig. 7. $y$-component velocity along the horizontal central line of lid-driven cavity.

Table 1

Average Nusselt number on the interface with various $k$.

\begin{tabular}{cccc}
\hline & $k=0.1$ & $k=1$ & $k=10$ \\
\hline Ref.[18] & 2.899 & 2.468 & 1.468 \\
present results & 2.9696 & 2.5303 & 1.4635 \\
\hline
\end{tabular}

Table 1 lists the comparison of the average Nusselt number $N u$ on the interface between fluid and solid bottom wall, which is calculated by $N u=$ $-\frac{1}{H} \int_{0}^{H} \frac{\partial T}{\partial y} d x$. There are only slight differences between the present numerical prediction and the published data [18].

Figure 8 illustrates the profiles of temperature and $x$-component velocity along the vertical central line of the investigated domain, with a comparison to the data obtained by the commercial CFD software FLUENT ${ }^{\circledR}$. The comparison shows the present model can capture the fine structures of flow and temperature field accurately.

\subsection{MHD mixed convection with Joule heating in a lid-driven cavity which contains a heat conducting horizontal circular cylinder}

Finally, the MHD mixed convection in a lid-driven cavity investigated in Ref.[19] is adopted to validate the present model's accuracy for modeling conjugate heat transfer with complicated interfaces. As shown by Fig. 9, a heat conducting horizontal circular cylinder, whose diameter is $d$, is located in the center of the cavity. The horizontal walls of the cavity are adiabatic. The temperature on the right vertical wall is $T_{h}=1$ while that on the left lateral wall is $T_{c}=0$. The left lateral wall is moving with velocity $v=1$ while the other walls are stationary. The gravity $g$ goes downwards. In addition, the working MHD 


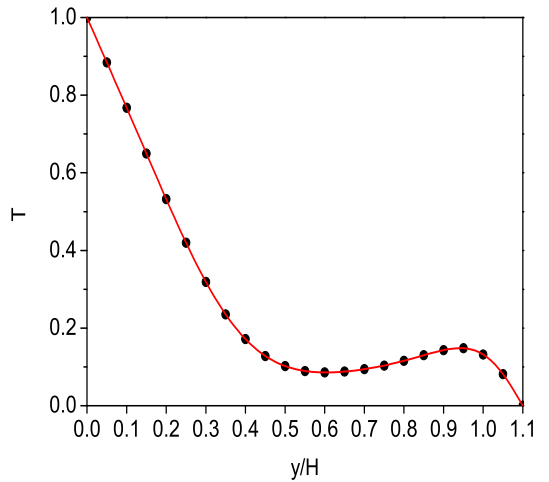

(a)

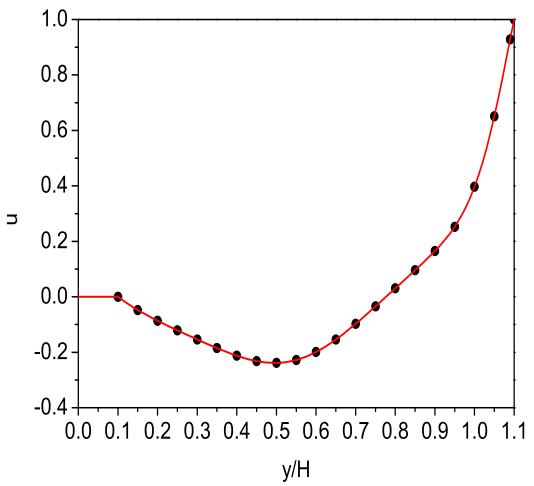

(b)

Fig. 8. Profiles of (a) temperature and (b) $x$-component velocity along the vertical central line of the investigated domain for $h / H=0.1, k=1$ and $R i=1$ : dots-CFD, lines-present results.

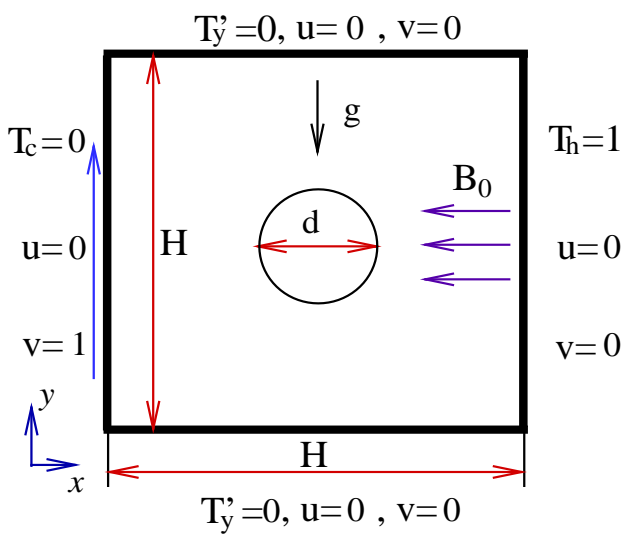

Fig. 9. Schematic configuration of MHD mixed convection with joule heating in lid-driven cavity containing a heat conducting horizontal circular cylinder.

fluid is subject to uniform external magnetic field $B_{0}$. Accordingly, the Joule heating should be considered, which acts like an internal heat source. In order to reflect the effect of Joule heating an additional term $J_{e} v^{2}$ should be added into the right hand of Eq. (1), where $J_{e}$ is the Joule heating parameter and $v$ is the $y$-component velocity. Therefore, a corresponding additional source term $\omega_{j} \Delta t J_{e} v^{2} / \sigma$ should be added into the LB evolving equation Eq.(8). The dimensionless parameters used in the present simulation are the Joule heating parameter $J_{e}=1$, the Richardson number $R i=1$, the Hartmann number $H a=10$ and the Reynolds number $R e=100$. The ratio of thermal conductivity between solid cylinder and MHD fluid $K=\lambda_{s} / \lambda_{f}$ varies from 1 to 10 . The diameter of the cylinder varies in the range $d / H=0.2$ and 0.6. The detailed description about the investigated domain and macroscopic governing equations please refer to Ref.[19]. A grid resolution $100 \times 100$ is adopted. 


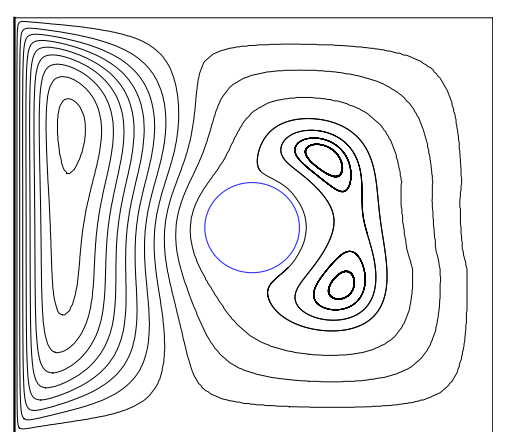

(a)

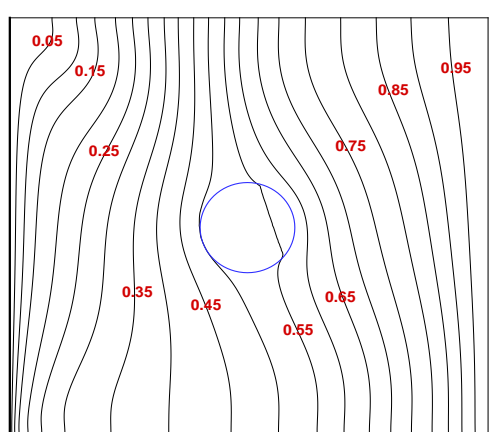

(b)

Fig. 10. (a) streamlines and (b)isotherms for $d / H=0.2$ and $K=5$.

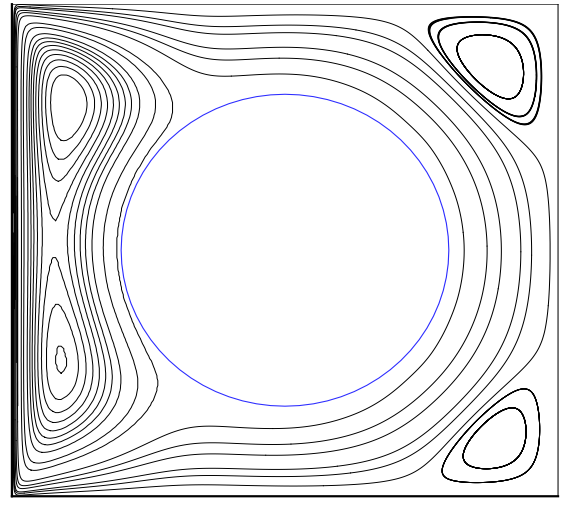

(a)

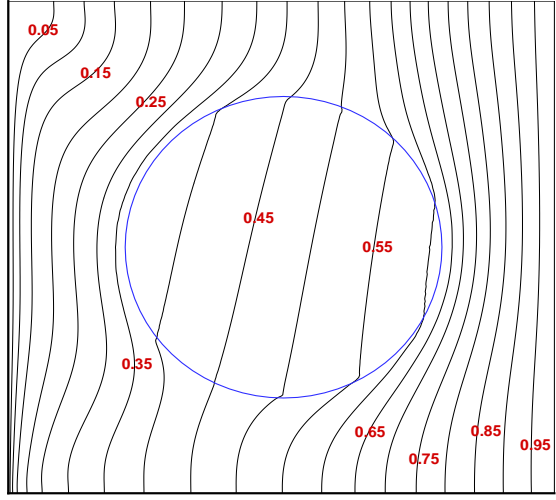

(b)

Fig. 11. (a) streamlines and (b)isotherms for $d / H=0.6$ and $K=5$.

Figures 10-11 plot the streamlines and isotherms when the size of the cylinder is changing while the solid-fluid thermal conductivity ratio is fixed at $K=5$. The influence of cylinder size on flow and temperature field is significant. With a small cylinder $(d / H=0.2)$, the fluid flow is featured by a pair of counter rotating rolls. While for a large cylinder $(d / H=0.6)$, the fluid flow is dominated by a clockwise vortex generated by the movement of the left vertical wall, together with twin counterclockwise small vortices caused by the buoyant force. The dramatic change of the flow field results from that a large cylinder can reduce the available space for the buoyancy-induced recirculation. The convective distortion of the isotherms near the right top corner of the enclosure decreases against $d$ increasing. The observation agrees well with that reported by Ref.[19].

Figure 12 illustrates the effect of $K$ on the distribution of isotherms. The corresponding streamlines are not plotted as the impact of $K$ on flow field is relatively slight and they are very similar with Fig.10 (a). According to this 


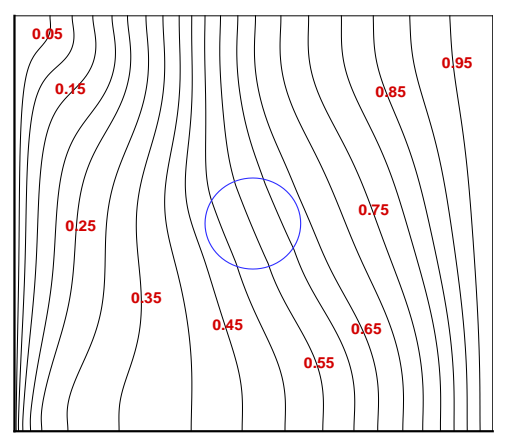

(a)

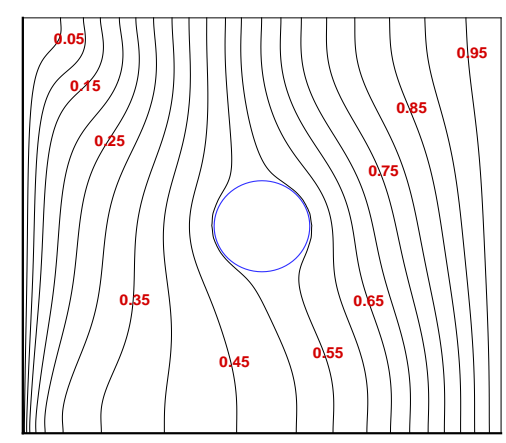

(b)

Fig. 12. Isotherms of (a) $K=10$ and (b) $K=10$ at $d / H=0.2$.

Table 2

Average Nusselt number on the left wall with various $d$ and $K=5$.

\begin{tabular}{ccc}
\hline & $d / H=0.2$ & $d / H=0.6$ \\
\hline Ref.[19] & 1.022651 & 1.547871 \\
present results & 1.082642 & 1.620414
\end{tabular}

Table 3

Average Nusselt number on the left wall with various $K$ and $d / H=0.2$.

\begin{tabular}{ccc}
\hline & $K=1$ & $K=10$ \\
\hline Ref.[19] & 0.981962 & 1.033299 \\
present results & 1.011489 & 1.105778 \\
\hline
\end{tabular}

figure, one can observe that the ratio of thermal conductivity between solid cylinder and fluid critically affects the distribution of isotherms. With a high $K$ (e.g. $K=10)$, the isotherms in the vicinity of the solid cylinder will be distorted significantly. The above phenomena were also reported by Ref.[19].

Tables 2-3 list the average Nusselt number $N u$ on the left wall at different configurations, compared with the open data in Ref.[19]. It can be found that $N u$ increases with $d$ and $K$, which is consistent with that reported by Ref.[19].

\section{Conclusion}

Conjugated heat transfer is a popular problem in industry. For traditional numerical tools, how to solve conjugated problems with complicated geometry is still a challenge. Recently, some scholars have conducted a number of efforts 
to develop LB-based approaches to address this challenge. Unfortunately, the shortcomings of these LB-based approaches are obvious, too. In the present work, we firstly analyze the advantages of the LB method for conjugate problems and reveal the root which restrict the standard LB method to model conjugate heat transfer. Based on the analyses, we propose a new LB model which can remedy the shortcomings of the available relevant LB models via a simple way. Some important advantages of the standard LB method are preserved. Any specific treatment dependent on interface topology is avoided by the present model. Moreover, it can be used for unsteady problems with complicated and time dependent interfaces and changeable thermophysical properties. The accuracy and reliability of the present model are validated by three nontrivial benchmark tests. The good agreements between the the present numerical prediction and available open data demonstrate the applicability of the present model for complicated conjugated heat transfer problems. Especially, the present model could be extended to some other important areas, such as fluid-solid phase change modeling.

Finally, although in the present study we only take a single-relaxation-time LB model as an example to show how to address the variation of thermophysical properties of working media, the extension to its multiple-relaxation-time counterpart is straightforward [8]. It will be considered in our future work.

\section{Acknowledgments}

This work has received funding from the Universidad Carlos III de Madrid, the European Unions Seventh Framework Programme for research, technological development and demonstration under grant agreement No. 600371, el Ministerio de Economa y Competitividad (COFUND2014-51509), el Ministerio de Educacin, cultura y Deporte (CEI-15-17) and Banco Santander. S. Chen would also acknowledge the support from the British Newton Alumni Fellowship Scheme.

\section{A Appendix: Derivation of Eq. (7)}

Replacing $T$ in Eq. (1) by $h^{*}$, one can obtain the following equation:

$$
\partial_{t} \sigma h^{*}+\nabla_{\alpha} \sigma h^{*} u_{\alpha}=\nabla_{\alpha} \lambda \nabla_{\alpha} \frac{h^{*}}{\left(\rho C_{p}\right)_{0}}
$$

The same as all previous research [5-9,11-13], at current stage we only consider the scenario where the heat capacitance varies spatially but keep constant over 
time. Consequently, the left hand side of Eq.(A.1) can be written as:

$$
\partial_{t} \sigma h^{*}+\nabla_{\alpha} \sigma h^{*} u_{\alpha}=\sigma\left(\partial_{t} h^{*}+\nabla_{\alpha} h^{*} u_{\alpha}\right)+h^{*} u_{\alpha} \nabla_{\alpha} \sigma+h^{*} \partial_{t} \vec{\sigma}^{0}
$$

With the aid of Eq.(A.2), Eq.(A.1) can be transformed to:

$$
\sigma\left(\partial_{t} h^{*}+\nabla_{\alpha} h^{*} u_{\alpha}\right)=\nabla_{\alpha} \lambda \nabla_{\alpha} \frac{h^{*}}{\left(\rho C_{p}\right)_{0}}-h^{*} u_{\alpha} \nabla_{\alpha} \sigma
$$

Dividing Eq.(A.3) by $\sigma$, there is:

$$
\partial_{t} h^{*}+\nabla_{\alpha} h^{*} u_{\alpha}=\frac{1}{\sigma} \nabla_{\alpha} \lambda \nabla_{\alpha} \frac{h^{*}}{\left(\rho C_{p}\right)_{0}}-\frac{h^{*}}{\sigma} u_{\alpha} \nabla_{\alpha} \sigma
$$

As

$$
\frac{1}{\sigma} \nabla_{\alpha} \lambda \nabla_{\alpha} \frac{h^{*}}{\left(\rho C_{p}\right)_{0}}=\nabla_{\alpha} \frac{\lambda}{\rho C_{p}} \nabla_{\alpha} h^{*}-\frac{\lambda}{\left(\rho C_{p}\right)_{0}} \nabla_{\alpha} h^{*} \nabla_{\alpha} \frac{1}{\sigma}
$$

With the aid of Eq.(A.5), Eq.(A.4) can be written as

$$
\partial_{t} h^{*}+\nabla_{\alpha} h^{*} u_{\alpha}=\nabla_{\alpha} \frac{\lambda}{\rho C_{p}} \nabla_{\alpha} h^{*}-\frac{\lambda}{\left(\rho C_{p}\right)_{0}} \nabla_{\alpha} h^{*} \nabla_{\alpha} \frac{1}{\sigma}-\frac{h^{*}}{\sigma} u_{\alpha} \nabla_{\alpha} \sigma
$$

namely Eq. (7).

If the heat capacitance varies over time, namely the last term of Eq.(A.2) $h^{*} \partial_{t} \sigma \neq 0$, it is also can be treated straightforwardly by the present scheme. What should be done is just to add $h^{*} \partial_{t} \sigma$ into the source term $S$ in Eq.(8). For the LB method, there is no obvious extra computational cost to evaluate $h^{*} \partial_{t} \sigma$ if the forward Euler time discretization scheme is adopted.

\section{References}

[1] Perelman T.L. On conjugated problems of heat transfer. International Journal of Heat and Mass Transfer 1961;3: 293-303.

[2] Chen X, Han P. A note on the solution of conjugate heat transfer problems using SIMPLE-like algorithms, Int. J. Heat Fluid Flow 2000;21: 463-467.

[3] Sato N, Takeuchi S, Kajishima T, Inagaki M, Horinouchi N. A consistent direct discretization scheme on Cartesian grids for convective and conjugate heat transfer. Journal of Computational Physics 2016;321:76-104

[4] Succi S. The lattice Boltzmann equation for fluid dynamics and beyond. Oxford: Oxford university press;2001.

[5] Wang J, Wang M, Li Z. A lattice Boltzmann algorithm for fluidCsolid conjugate heat transfer. International Journal of Thermal Sciences 2007;46: 228-234. 
[6] Meng F, Wang M, Li Z. Lattice Boltzmann simulations of conjugate heat transfer in high-frequency oscillating flows. International Journal of Heat and Fluid Flow 2008;29:1203-1210

[7] Li L, Chen C, Mei R, Klausner JF. Conjugate heat and mass transfer in the lattice Boltzmann equation method. Physical Review E 2014;89:043308/1043308/21.

[8] Le G, Oulaid O, Zhang JF. Counter-extrapolation method for conjugate interfaces in computational heat and mass transfer. Physical Review E 2015;91:033306/1-033306/11

[9] Hu Y, Li D, Shu S, Niu X. Full Eulerian lattice Boltzmann model for conjugate heat transfer. Physical Review E 2015;92:063305/1-063305/12

[10] Yang B, Chen S, Cao C, Liu Z, Zheng C. Lattice Boltzmann simulation of two cold particles settling in Newtonian fluid with thermal convection. International Journal of Heat and Mass Transfer 2016;93:477-490

[11] Karani H, Huber C. Lattice Boltzmann formulation for conjugate heat transfer in heterogeneous media. Physical Review E 2015;91:023304/1-023304/10.

[12] Rihab H, Moudhaffar N, Sassi BN, Patrick P. Enthalpic lattice Boltzmann formulation for unsteady heat conduction in heterogeneous media. International Journal of Heat and Mass Transfer 2016;100: 728-736

[13] Huang R, Wu H. Total enthalpy-based lattice Boltzmann method with adaptive mesh refinement for solid-liquid phase change. Journal of Computational Physics 2016; 315:65-83

[14] He XY, Luo LS. Lattice Boltzmann model for the incompressible Navier-Stokes equation. Journal of Statistical Physics 1997;88:927-944.

[15] Chen S, Liu Z, Zhang C, He Z, Tian Z, Shi B, Zheng C. A novel coupled lattice Boltzmann model for low Mach number combustion simulation. Applied Mathematics and Computation 2007;193 : 266-284.

[16] Chen S, Luo K, Zheng C. A simple enthalpy-based lattice Boltzmann scheme for complicated thermal systems. Journal of Computational Physics 2012;231:82788294 .

[17] Chatterjee D, Chakraborty S. An enthalpy-based lattice Boltzmann model for diffusion dominated solid-liquid phase transformation. Physics Letters A 2005;341:320-330

[18] Oztop HF, Sun C, Yu B. Conjugate-mixed convection heat transfer in a liddriven enclosure with thick bottom wall. International Communications in Heat and Mass Transfer 2008;35: 779-785

[19] Rahman MM, Mamun MAH, Saidur R, Nagata S. Effect of a heat conducting horizontal circular cylinder on MHD mixed convection in a lid-driven cavity along with joule heating. International Journal of Mechanical and Materials Engineering 2009;4:256-265 